\section{Imaging aspect of neuromyelitis optica: a case report and review of the literature}

\author{
H Lhajoui*, I Bounnit, N Moussali, A Merzem, O Amriss, \\ $\mathrm{H}$ Belgadir and N Elbenna
}

Department of Radiology, Ibn Rochd University Hospital Center of Casablanca, Radiology Service of August 20, 1953 Hospital, Morocco

\section{Abstract}

The case report presents a neuromyelitis optica in a 19 years old male. Brain and spinal cord MRI showed bilateral optic neuropathy, multiphasic demyelinating process involving the cervical and thoracic spinal cord. Cerebrospinal fluid showed negative NMO Ig G. We will describe the radiological aspect of neuromyelitis optica with a review of the literature.

\section{Introduction}

Neuromyelitis optica or Devic's disease (NMOSD), is a rare demyelinating disorder of the central nervous system that has a prédilection for the optic nerve and spinal cord. The diagnosis of NMO requires the following criteria: presence of optic neuritis, myelitis, involvement of spinal cord lesions in 3 or more segments by magnetic resonance imaging.

\section{Case presentation}

We report a case of a 19 years old male, who presents a tetraparesis with bilateral blindness progressing for 5 months, aggravated by respiratory tip. Brain and spinal cord MRI showed bilateral optic neuropathy, signal hyperintensities at medulla oblongata, cervical and thoracic spinal cord on the T2 weighted sequence, spans over 3 contiguous vertebral, not enhanced by the injection of Gadolinium. Cerebrospinal fluid showed negative NMO Ig G. Finally, he was diagnosed with NMOSD and received methylprednisolone treatment. The evolution was favorable with disappearance of the symptoms (Figures 1,2).

\section{Discussion}

Neuromyelitis optica spectrum disorder (NMOSD) is an uncommon antibody-mediated disease of the central nervous system with a prédilection for the spinal cord and optic nerves.

The term neuromyelitis optica: NMO (derived from neuromyélite optique aiguë) was first described by Eugène Devic and his doctoral student Fernand Gault in 1894 [1]. The disease
More Information

*Address for Correspondence: Lhajoui $\mathrm{H}$, Department of Radiology, Ibn Rochd University Hospital Center of Casablanca, Radiology Service of August 20, 1953 Hospital, Morocco, Email: hayatt.Ihajoui@gmail.com

Submitted: September 06, 2021 Approved: December 06, 2021 Published: December 07, 2021

How to cite this article: Lhajoui $\mathrm{H}$, Bounnit I, Moussali N, Merzem A, Amriss O, et al. Imaging aspect of neuromyelitis optica: a case report and review of the literature. Arch Case Rep. 2021; 5: 034-036.

DOI: 10.29328/journal.acr.1001055

Copyright License: (C) 2021 Lhajoui $\mathrm{H}$, et al. This is an open access article distributed under the Creative Commons Attribution License, which permits unrestricted use, distribution, and reproduction in any medium, provided the original work is properly cited.

Keywords: Neuromyelitis; Neuropathy; Optic

\section{Check for updates}

OPEN ACCESS

was therefore previously referred to as Devic's disease. Until recently it was unclear whether neuromyelitis optica was a separate disease or merely a more severe form of opticospinal multiple sclerosis (MS). Since the discovery in 2005 of the AQP4 antibody (AQP4-Ab) biomarker associated with $70 \%-80 \%$ of cases [2], NMOSD can be reliably distinguished from multiple sclerosis (MS).
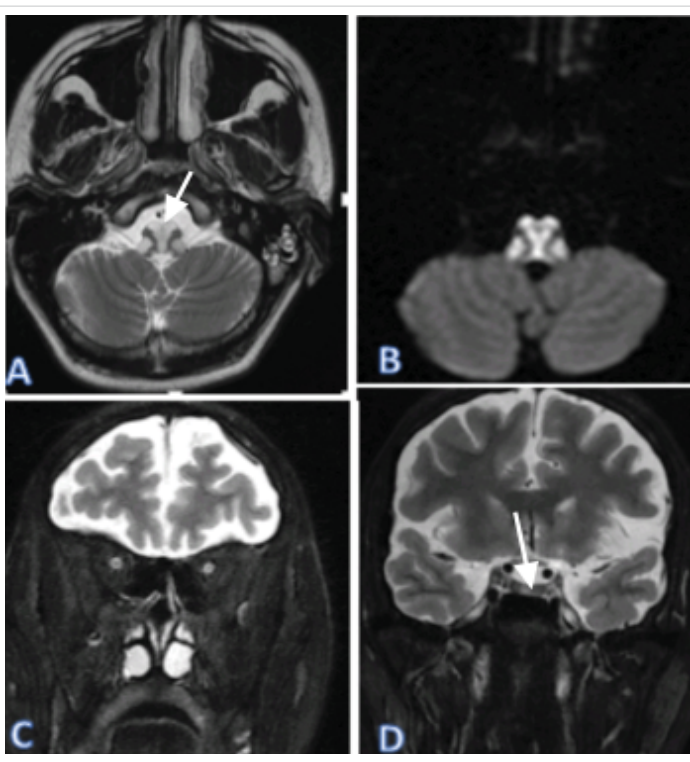

Figure 1: Brain MRI on a axial (A, B), coronal (C, D) 2-weighted image $(C)$, showed bilateral signal hyperintensities at medulla oblongata and bilateral optic nerve on T2. 


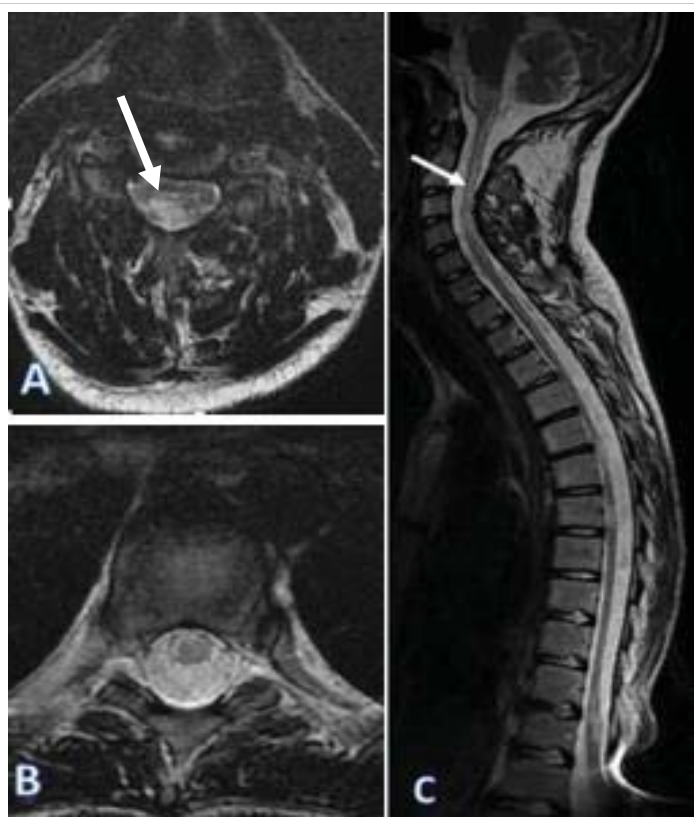

Figure 2: Spinal Cord MRI on a axial $(A, B)$, sagittal $(C)$, showed signal hyperintensities at cervical and thoracic spinal cord on the T2 weighted sequence, spans over 3 contiguous vertebral.

MRI has an important rôle in differentiating NMOSD from other inflammatory disorders of the CNS, particularly from MS. differentiating these conditions is critical because treatments are distinct.

\section{Optic nerve and chiasm}

Studies have reported non-specific optic nerve sheath thickening, optic nerve hyperintensities on T2- weighted sequences, and gadolinium enhancement on T1-weighted sequences in acute optic nerve of NMO. To differentiate the NMOSD from MS, typical features of optic neuritis were demonstrated: Bilateral and longitudinally extensive optic nerve involvement, usually affecting more than half of the length of the optic nerve, optic neuritis preferentially compromises the posterior optic pathway and typically includes the intracranial segment of the optic nerves, usually extending to the chiasm and optic tract. In chronic stages, atrophy of the optic nerves and variable hyperintensity on T2weighted images are frequently observed $[3,4]$.

\section{Spinal cord}

The inflammatory process of NMOSD in spinal cord MRI is characterized by hyperintensity on T2- weighted sequences and by hypointensity on T1- weighted sequences. These abnormalities are more frequently present in the cervical and the upper thoracic spinal cord segments than the lower thoracic and lumbar regions with a preferential involvement in the central gray matter. In the spinal cord, AQP4 is abundant in the gray matter and in glial cell processes adjacent to the ependymal cells of the central canal and to a lesser degree in the white matter of the spinal cord.

The most distinct manifestation of NMO is longitudinally extensive spinal cord lesion (LETM), defined as a lesion that spans over 3 or more contiguous vertebral segments and predominantly involves central gray matter on the spinal cord MRI . LETM seems to be less specific for NMO in children than in adults. Chronic lesions can be short or replaced by long segments of atrophy or myelomalacia (pseudosyrinx) $[4,5]$.

Two of the most typical MR imaging features that assist NMOSD diagnosis are bright spotty lesions on T2-weighted images (higher than CSF) and corresponding dark lesions on T1-weighted images [6].

In acute phases, cord swelling and irregular enhancement on T1-weighted images can be demonstrated, usually appearing as a combination of hypointense patchy areas and ring-enhancing lesions [6].

\section{Brain}

Although NMO was thought to have normal intracranial appearance, the prevalence of asymptomatic abnormalities is increasing in the majority of seropositive NMOSD patients. To recognize typical brain lesions in NMOSD, one must primarily remember the areas where AQP4 is consistently expressed, that is, the subpial regions, periependymal regions, circumventricular organs, brainstem, chiasm/hypothalamus, and corpus callosum. Confluent hyperintensities on FLAIR/ T2-weighted images have been reported that are usually asymmetrically distributed in periependymal areas, following the ependymal lining of the lateral, third, and fourth ventricles, particularly close to the cerebral aqueduct. The ependymal surfaces of the corpus callosum, diencephalic region, and brainstem have also been assumed as typical locations for NMOSD brain lesions.

The corpus callosum is typically involved at its ependymal surface, with the involvement of-ten affecting most of its length. In acute phases, edematous and heterogeneous hyperintensity on FLAIR/T2-weighted images is usually observed, assuming a typical marbled or "arch bridge" appearance $[3,4]$.

Tumefactive brain lesions ( $>3 \mathrm{~cm}$ in longest diameter) may be present in NMOSD, par- ticularly in AQP4-IgG-seropositive patients. However, this MR imaging finding is more common in multiple sclerosis patients [3].

\section{Conclusion}

Neuromyelitis optica spectrum disorder (NMOSD) is a relapsing central nervous system disease associated with aquaporin-4 antibodies. Common presentations include longitudinally extensive myelitis, severe optic neuritis, and area postrema syndrome.

\section{References}

1. Jarius S, Wildemann B. The history of neuromyelitis optica. J Neuroinflammation. 2013; 10: 8.

PubMed: https://pubmed.ncbi.nlm.nih.gov/23320783/

2. Huda S, Whittam D, Bhojak M, Chamberlain J, Noonan C, et al. 
Neuromyelitis optica spectrum disorders. Clin Med. 2019; 19: 169-176. PubMed: https://pubmed.ncbi.nlm.nih.gov/30872305/

3. Dutra BG, da Rocha AJ, Nunes RH, Maia ACM Jr,. Neuromyelitis Optica Spectrum Disorders: Spectrum of MR Imaging Findings and Their Differential Diagnosis. Radiographics. 2018; 38: 169-193. PubMed: https://pubmed.ncbi.nlm.nih.gov/29320331/

4. Kim HJ, Paul F, Lana-Peixoto, Tenembaum $\mathrm{S}$, Asgari $\mathrm{N}$, et al. MRI characteristics of neuromyelitis optica spectrum disorder: An international update. Neurology. 2015; 84: 1165-1173.

PubMed: https://pubmed.ncbi.nlm.nih.gov/25695963/
5. Wingerchuk DM, Banwell B, Bennett JL, Cabre P, Carroll W, et al. International consensus diagnostic criteria for neuromyelitis optica spectrum disorders. Neurology. 2015; 85: 177-189.

PubMed: https://pubmed.ncbi.nlm.nih.gov/26092914/

6. Pekcevik $\mathrm{Y}$, Mitchell $\mathrm{CH}$, Mealy MA, Orman $\mathrm{G}$, Lee $\mathrm{IH}$, et al. Differentiating neuromyelitis optica from other causes of longitudinally extensive transverse myelitis on spinal magnetic resonance imaging. Mult Scler. 2015; 22: 302-311.

PubMed: https://pubmed.ncbi.nlm.nih.gov/26209588/ 